\title{
DESGASTE DE LA HERRAMIENTA DE CORTE EN EL TORNEADO EN SECO DEL ACERO AISI 316L
}

\section{WEAR OF THE CUTTING TOOL IN TURNING DRY STEEL AISI 316L}

\author{
Yoandrys Morales-Tamayo ${ }^{1, *}$, Yusimit Zamora-Hernández ${ }^{2}$, Roberto Félix \\ Beltrán-Reyna $^{3}$, Kimberly Magaly López-Cedeño ${ }^{4}$, Ringo John López-Bustamante ${ }^{5}$
}

\section{Resumen}

El presente estudio experimental se centra en determinar modelos estadísticos para predecir el desgaste del flanco durante el torneado en seco del acero inoxidable AISI 316L utilizando dos insertos de corte. Los parámetros de corte fueron comparados utilizando un análisis de varianza y de regresión múltiple. Como principales resultados se obtuvieron el efecto significativo del avance de corte y el tiempo principal en el desgaste del flanco. Se hallaron los modelos estadísticos para estimar el desgaste de flanco, así como los errores absolutos medios de cada ecuación. El mejor desempeño correspondió al inserto GC2015 según las ecuaciones de regresión.

Palabras clave: torneado en seco, desgaste del flanco, acero inoxidable AISI 316L, análisis de varianza y regresión.

\begin{abstract}
The present experimental study focuses on determining statistical models to predict flank wear during dry turning of AISI 316L stainless steel using two cutting inserts. Cutting parameters were compared using analysis of variance and simple regression. As a main result the significant effect of cutting feed and main time was found. Statistical models were used to estimate flank wear, as well as the mean absolute errors of each equation. The best performance corresponded to the GC2015 insert according to the regression equations.
\end{abstract}

Keywords: Dry turning, Flank wear, AISI 316L Stainless steel, Analysis of variance and regression.

\footnotetext{
${ }_{1, *}$ Coordinación de Investigación, Universidad Técnica de Cotopaxi Extensión La Maná, Cotopaxi - Ecuador. Autor para correspondencia yoandrys.morales@utc.edu.ec

${ }^{2}$ Departamento de Ingeniería Mecánica, Universidad de Granma, Bayamo - Cuba.

${ }^{3}$ Departamento de Energía y Mecánica, Universidad de las Fuerzas Armadas, Cotopaxi - Ecuador.

${ }^{4}$ Facultad de Química, Universidad de Guayaquil - Ecuador.

${ }^{5}$ Universidad Técnica de Cotopaxi, Extensión La Maná, Cotopaxi - Ecuador.
}

Recibido: 21-11-2016, aprobado tras revisión: 20-12-2016

Forma sugerida de citación: Morales, Y.; Zamora, Y.; Beltrán, R; López, K.; López, R. (2017). «Desgaste de la herramienta de corte en el torneado en seco del acero AISI 316L». INGENIUS. N. ${ }^{\circ} 17$, (Enero-Junio). pp. 36-41. ISSN: 1390-650X. 


\section{Introducción}

Las industrias dedicadas a la manufactura de elementos mecánicos están constantemente esforzándose para reducir costos e incrementar la calidad de las piezas maquinadas teniendo en cuenta la creciente demanda de productos con una elevada precisión dimensional.

El acero constituye un material básico para el desarrollo, debido a sus propiedades versátiles y posibilidades de reciclado. El acero se aplica en casi todos los sectores industriales importantes dedicados a la fabricación de piezas [1].

En los últimos tiempos, debido a la necesidad de materiales para herramientas de corte cada vez más resistentes al desgaste han aparecido materiales avanzados que revoluciona el corte de metales; sustentados en la aparición de recubrimientos de pequeños espesores y en los procesos de difusión térmica [2].

Muchas soluciones para aumentar la vida de la herramienta han estado dirigidas a disminuir la temperatura en la interface herramienta-pieza mediante la utilización y desarrollo de fluidos de corte de diferentes naturalezas. La utilización de estos encarece los costos de fabricación, provoca daños en la salud de los operarios y afecta el medioambiente [3]. Las nuevas tecnologías para atenuar los efectos perjudiciales de los fluidos de corte se sustentan en el corte en seco, en la mínima lubricación y en el desarrollo de nuevas fórmulas de fluidos [4].

El mecanizado en seco se considera como la mejor tecnología para eliminar el uso de los fluidos de corte en las empresas manufactureras y así reducir los costos de mecanizado y los riesgos ecológicos [5]. Esta investigación tiene como objetivo determinar modelos estadísticos para predecir el desgaste del flanco durante el torneado en seco del acero inoxidable AISI 316L.

\section{Materiales y métodos}

\subsection{Materiales}

El material utilizado correspondió al acero inoxidable austenítico AISI 316L, acero utilizado en la manufactura de equipos para procesos químicos y alimenticios, en la industria aeroespacial, en la automotriz, en la biomédica para implantes médicos [6,7]. La composición química del AISI 316L se puede observar en la Tabla 1, para ello se utilizó un espectrómetro de emisión de chispa.

Tabla 1. Composición química de la probeta

\begin{tabular}{ccccccccc}
\hline $\mathbf{C}$ & $\mathbf{S i}$ & $\mathbf{M n}$ & $\mathbf{C r}$ & $\mathbf{M o}$ & $\mathbf{N i}$ & $\mathbf{P}$ & $\mathbf{S}$ & $\mathbf{N}$ \\
\hline 0,015 & 0,58 & 1,5 & 16,95 & 2,05 & 10,08 & 0,031 & 0,029 & 0,059 \\
\hline
\end{tabular}

Las herramientas de corte correspondieron a insertos recubiertos, de marca Sandvik PVD GC1115 y
$C V D$ GC2015. La geometría seleccionada para los dos insertos fue $C C M T 120404-M F$ con rompevirutas. Esta plaquita es rómbica de $80^{\circ}$ positiva, con un ángulo de incidencia $\alpha p=7^{\circ}$, espesor $s=4,76 \mathrm{~mm} \pm 0,13 \mathrm{~mm}$, círculo inscrito $i C=12,7 \mathrm{~mm} \pm 0,08 \mathrm{~mm}$. Es una plaquita tipo $\mathrm{T}$, con una longitud de la arista de corte $l=12 \mathrm{~mm}$, radio de la punta $r_{\varepsilon}=0,4 \mathrm{~mm}$ y propuesta para operaciones de acabado de los aceros inoxidables austeníticos (MF) [8].

Se determinó el tipo y espesor de los recubrimientos utilizando el microscopio electrónico de barrido marca JEOL. Del mismo modo, se estableció la composición química de los insertos (Tabla 2).

Tabla 2. Objetivos y rango de operación del eje Y.

\begin{tabular}{ccc}
\hline Parámetros & Insertos & \\
Recubrimientos & GC1115 & GC2015 \\
\hline Primera capa & $\mathrm{TiN}$ & $\mathrm{TiN}$ \\
Segunda capa & - & $\mathrm{Al} 2 \mathrm{O} 3$ \\
Tercera capa & - & $\mathrm{Ti}(\mathrm{N}, \mathrm{C})$ \\
Espesor $(\mu \mathrm{m})$ & 5 & 15 \\
\hline Sustratos $(\%)$ & GC1115 & GC2015 \\
\hline $\mathrm{W}$ & 94,67 & 96,35 \\
$\mathrm{Ti}$ & 2,23 & 1,57 \\
$\mathrm{Co}$ & 3,1 & 2,08 \\
\hline
\end{tabular}

\subsection{Equipos}

Las pruebas durante el torneado fueron desarrolladas en un torno CNC Okuma modelo Multus B-200W. Para evitar confusión y errores entre las muestras se utilizó un borde de la herramienta de corte para cada ensayo. El criterio de desgaste adoptado de la Norma ANSI/ SME B94.55M fue de $200 \mu \mathrm{m}$ [9].

El análisis factorial completo fue el procedimiento utilizado para determinar la relación entre las variables independientes (parámetros de corte) y la variable dependiente (desgaste del flanco $(w)$ ). Un total de 64 ensayos para dos réplicas fueron desarrollados con dos niveles de velocidades de corte $(v)$, cuatro niveles de tiempo $(T)$, dos niveles de avances de corte $(f)$ y dos niveles de material de la herramienta, en la Tabla 3 aparecen las variables estudiadas.

Tabla 3. Variables consideradas en el estudio.

\begin{tabular}{clcc}
\hline $\mathbf{v}(\mathbf{m} / \mathbf{m i n})$ & $\mathbf{f}(\mathbf{m m} / \mathbf{r e v})$ & $\mathbf{T}(\mathbf{m i n})$ \\
\hline 400 & 0,1 & 0,2 & $2 ; 3 ; 4 ; 5$ \\
450 & 0,1 & 0,2 & 0,$6 ; 1,2 ; 2 ; 3$ \\
\hline
\end{tabular}

Luego fue desarrollado un análisis de regresión múltiple para construir los modelos estadísticos que describen el impacto de los dos factores estudiados, el tiempo de mecanizado y el avance de corte en el desgaste del flanco. El estado de los instrumentos de medidas, las máquinas y el personal de experimentación fue 
comprobado mediante pruebas iniciales. El desgaste del flanco de la herramienta de corte fue medido con un microscopio electrónico de barrido marca JEOL.

\section{Resultados y discusión}

En las Figuras 1 a la 4 se muestran las mediciones del desgaste del flanco de los insertos para $400 \mathrm{~m} / \mathrm{min}$ y $450 \mathrm{~m} / \mathrm{min}$ de velocidad de corte, para 5 y 3 min de tiempo de maquinado respectivamente. Se observa que para $v=400 \mathrm{~m} / \mathrm{min}$ y $f=0,16 \mathrm{~mm} / \mathrm{rev}$ los insertos GC1115 y GC2015 no exceden el criterio de fin de vida del desgaste del flanco $(200 \mu \mathrm{m})$. Para la velocidad de $450 \mathrm{~m} / \mathrm{min}$ y avance $0,16 \mathrm{~m} / \mathrm{min}$ el inserto GC1115 sufre un desgaste excesivo sobrepasando el criterio de fin de vida del desgaste del flanco y el inserto GC2015 se aproxima a este límite, aunque no lo sobrepasa.

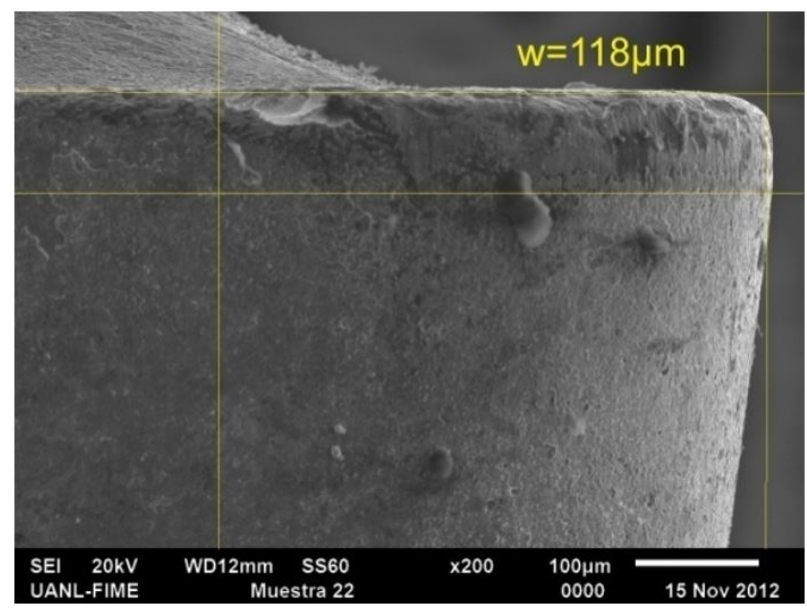

Figura 1. Desgaste del flanco de los insertos GC1115 para $\mathrm{v}=400 \mathrm{~m} / \mathrm{min}$ y $\mathrm{f}=0,16 \mathrm{~mm} / \mathrm{rev}$, durante $5 \mathrm{~min}$ de corte.

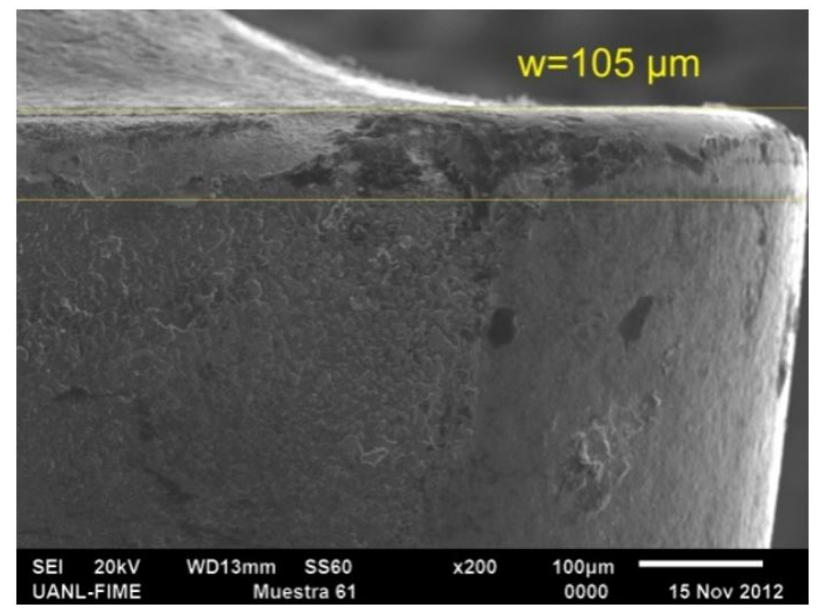

Figura 2. Desgaste del flanco de los insertos GC2015 para $\mathrm{v}=400 \mathrm{~m} / \mathrm{min}$ y $\mathrm{f}=0,16 \mathrm{~mm} / \mathrm{rev}$, durante $5 \mathrm{~min}$ de corte.

La utilización de elevadas velocidades de corte provoca un aumento de la temperatura en la herra- mienta de corte, como consecuencia debilita la arista de corte [10].

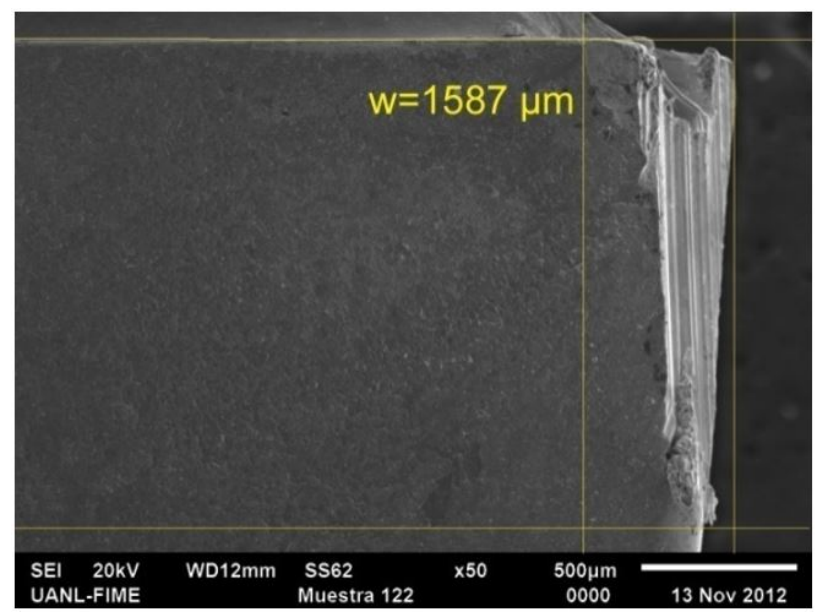

Figura 3. Desgaste del flanco de los insertos GC1115 para $\mathrm{v}=450 \mathrm{~m} / \mathrm{min} \mathrm{y} \mathrm{f}=0,16 \mathrm{~mm} / \mathrm{rev}$, durante 3 min de corte.

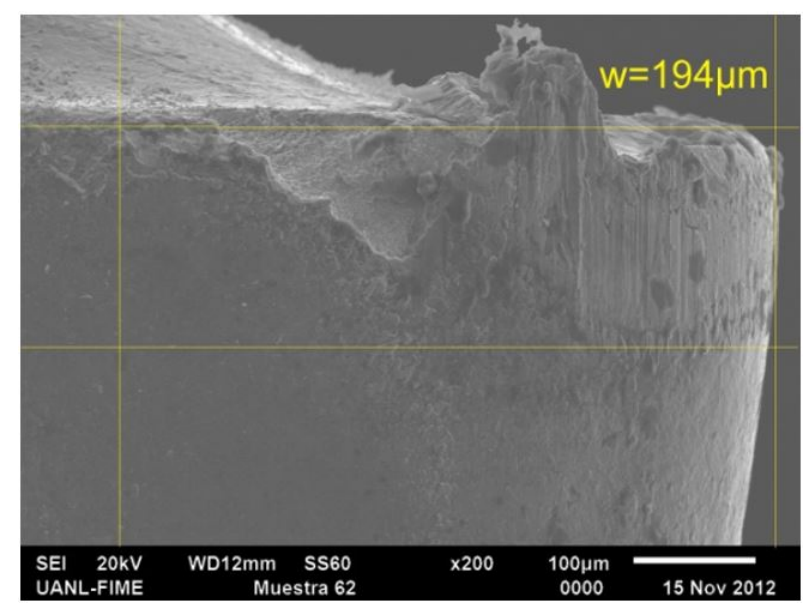

Figura 4. Desgaste del flanco de los insertos GC2015 para $\mathrm{v}=450 \mathrm{~m} / \mathrm{min} \mathrm{y} \mathrm{f}=0,16 \mathrm{~mm} / \mathrm{rev}$, durante 3 min de corte.

Los insertos de corte muestran desgaste por abrasión, adhesión, difusión y fractura del borde cortante, similar resultado obtenido por Jianxin y otros [11].

En la Figura 5a se muestra la evolución del desgaste del flanco de los insertos para $v=400 \mathrm{~m} / \mathrm{min}$, se aprecia un mejor comportamiento del inserto GC1115 hasta $4 \mathrm{~min}$ de corte. Para los $5 \mathrm{~min}$ de maquinado el inserto GC2015 obtuvo un mejor resultado en cuanto al desgaste, ninguno de los dos insertos alcanzó el límite del criterio de fin de vida (Figura 5a).

Para la velocidad de corte de $450 \mathrm{~m} / \mathrm{min}$ (Figura 5b) el mejor desempeño hasta 1,2 min de corte fue también para el inserto GC1115; en cambio para los tiempos restantes el desgaste del flanco en sus filos de corte fue superior hasta colapsar antes de alcanzar el tiempo final de mecanizado. 


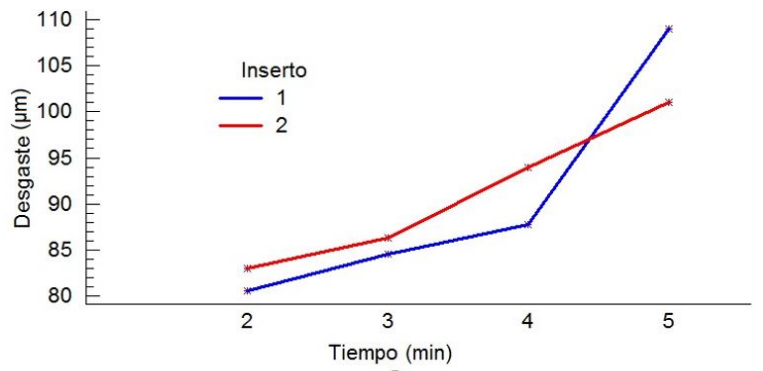

a

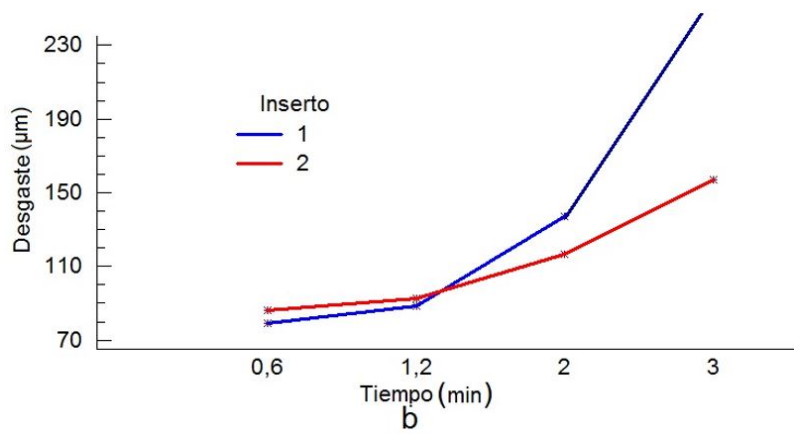

Figura 5. Evolución del desgaste del flanco de los insertos (a) para $400 \mathrm{~m} / \mathrm{min}$ y (b) $450 \mathrm{~m} / \mathrm{min}$.

En la investigación se encontró que la herramienta de menor dureza GC2015 (1404 HV) fue coincidentemente la de menor desgaste, debido a que posee tres recubrimientos específicos para mejorar la mecánica del corte. La capa compuesta por $\mathrm{Ti}(\mathrm{N}, \mathrm{C})$ le proporciona resistencia al desgaste y estabilidad térmica, la capa de $\mathrm{Al}^{2} \mathrm{O}^{3}$ le suministra resistencia al calor y disminuye el desgaste cráter y el recubrimiento de TiN le aporta resistencia al calor [12]. Además, la herramienta de mayor dureza solo presenta un recubrimiento $5 \mu \mathrm{m}$ de espesor mientras que la suma de los recubrimientos del inserto de tres capas alcanza $15 \mu \mathrm{m}$ de espesor.

$\mathrm{El}$ análisis de varianza fue realizado para determinar los factores estadísticamente significativos y las interacciones en el desgaste de flanco y, además, se realizó el análisis de regresión múltiple utilizando el software STATGRAPHICS Plus versión 5.1.

\subsection{Análisis de varianza y de regresión múlti- ple}

El análisis de varianza realizado en este estudio se puede observar en las Tablas 4 y 5 respectivamente. En ellas se muestran la contribución de cada factor y la importancia estadística para un $95 \%$ de confianza. En la investigación fueron significativos el avance de corte y el tiempo principal de corte, similar conclusión determinada por Galanis y Manolakos [13] y Hamdan [14].

Se realizó un análisis de regresión múltiple para describir la relación entre el desgaste del flanco, el tiempo de corte principal y el avance de corte para cada velocidad e inserto utilizados en el estudio. El propósito fue obtener los modelos estadísticos para predecir el desgaste del flanco durante el torneado en seco del acero inoxidable AISI 316L. Además, se observa el coeficiente de determinación $\left(\mathrm{R}^{2}\right)$ que representa la bondad de ajuste y el error absoluto medio (MAE).

Tabla 4. Análisis de varianza factorial del desgaste del flanco para $\mathrm{v}=400 \mathrm{~m} / \mathrm{min}$.

\begin{tabular}{cccccc}
\hline Fuente & $\begin{array}{c}\text { S. de } \\
\text { cuadrado }\end{array}$ & $\begin{array}{c}\text { Grado } \\
\text { libertad }\end{array}$ & C. medio & Co.-F & p-v \\
\hline Avance & 318,7 & 1 & 318.781 & 27.64 & 0.0001 \\
Inserto & 5,2 & 1 & 5,28125 & 0,46 & 0,508 \\
Tiempo & 2841,5 & 3 & 947,198 & 82,14 & 0 \\
Residuo & 445,8 & 26 & 17,1466 & & \\
Total & 3611.4 & 31 & & & \\
\hline
\end{tabular}

Tabla 5. Análisis de varianza factorial del desgaste del flanco para $\mathrm{v}=450 \mathrm{~m} / \mathrm{min}$.

\begin{tabular}{cccccc}
\hline Fuente & $\begin{array}{c}\text { S. de } \\
\text { cuadrado }\end{array}$ & $\begin{array}{c}\text { Grado } \\
\text { libertad }\end{array}$ & C. medio & Co.-F & p-v \\
\hline Avance & 273430 & 1 & 273430 & 8,96 & 0,091 \\
Inserto & 245350 & 1 & 245350 & 2,77 & 0,108 \\
Tiempo & 989653 & 3 & 329884 & 3,72 & 0,024 \\
Residuo & $2,3 \times 106$ & 26 & 88717,8 & & \\
Total & $3,8 \times 106$ & 31 & & & \\
\hline
\end{tabular}

En las tablas 6 y 7 se muestran las ecuaciones del modelo ajustado, el error absoluto medio y el coeficiente de determinación para las diferentes velocidades de corte utilizadas teniendo en cuenta diferentes tipos de modelos.

Los modelos con menores errores absolutos medios correspondieron al tipo exponencial con el inserto GC1115 y al tipo cúbico con el GC2015 durante el mecanizado con la velocidad de $400 \mathrm{~m} / \mathrm{min}$.

Por otro lado, ambos insertos alcanzaron los menores valores de errores absolutos medios con el modelo del tipo exponencial cuando fueron mecanizados con la velocidad de $450 \mathrm{~m} / \mathrm{min}$.

\section{Conclusiones}

El desgaste del flanco aumentó con el tiempo de maquinado hasta un valor máximo de $1587 \mu \mathrm{m}$ para las velocidades de corte utilizadas en el estudio. Las ecuaciones de regresión múltiple obtenidas para cada inserto de corte utilizado en este experimento permitieron determinar el tiempo de vida útil teniendo en cuenta el criterio de fin de vida. El mejor desempeño corresponde al inserto GC2015 con tiempos de vida útil de 8,64 min superiores al inserto GC1115 $(7,89 \mathrm{~min})$ con la velocidad de $400 \mathrm{~m} / \mathrm{min}$, al igual que para la otra velocidad donde el inserto GC2015 alcanzó un valor de 3.32 min y el GC1115 solo 0,73 min. 
Tabla 6. Análisis de regresión del desgaste del flanco para los insertos para $\mathrm{v}=400 \mathrm{~m} / \mathrm{min}$.

\begin{tabular}{lllccc}
\hline Inserto & \multicolumn{1}{c}{ Tipo } & \multicolumn{1}{c}{ Ecuación del modelo ajustado } & MAE & $\mathbf{R}^{2}$ \\
\hline \multirow{4}{*}{ GC1115 } & Lineal & $\mathrm{w}=50,125+81,25 \times \mathrm{f}+9,0 \times \mathrm{T}$ & 4,76 & 75,81 \\
& Polinomial & $\mathrm{w}=63,625+1,33333 \times \mathrm{T}^{2}+81,25 \times \mathrm{f}$ & 4,01 & 79,45 \\
& Cúbica & $\mathrm{w}=68,0659+0,242127 \times \mathrm{T}^{3}+81.26 \times \mathrm{f}$ & 3,57 & 84,26 \\
& Exponencial & $\mathrm{w}=80,3761+1,59068 \times e^{(\mathrm{T})} \times \mathrm{f}$ & 2,81 & 92,21 \\
\hline \multirow{6}{*}{ GC2015 } & Lineal & $\mathrm{w}=58,4125+7,025 \times \mathrm{T}+76,5625 \times \mathrm{f}$ & 2,28 & 89,87 \\
& Polinomial & $\mathrm{w}=69,4119+1,00653 \times \mathrm{T}^{2}+76,5625 \times \mathrm{f}$ & 1,94 & 91,3 \\
& Cúbica & $\mathrm{w}=73,07+0,177321 \times \mathrm{T}^{3}+76,5625 \times \mathrm{f}$ & 1,86 & 91,91 \\
& Exponencial & $\mathrm{w}=84.498+1.11207 \times e^{(\mathrm{T})} \times \mathrm{f}$ & 2,7 & 84,79 \\
\hline
\end{tabular}

Tabla 7. Análisis de regresión de la rugosidad superficial para los insertos para $\mathrm{v}=450 \mathrm{~m} / \mathrm{min}$.

\begin{tabular}{lllccc}
\hline Inserto & \multicolumn{1}{c}{ Tipo } & \multicolumn{1}{c}{ Ecuación del modelo ajustado } & MAE & $\mathbf{R}^{2}$ \\
\hline \multirow{4}{*}{ GC1115 } & Lineal & $\mathrm{w}=-764,171+312,454 \times \mathrm{T}+4346,88 \times \mathrm{f}$ & 274 & 41,42 \\
& Polinomial & $\mathrm{w}=-571,863+91,5845 \times \mathrm{T}^{2}+4346,88 \times \mathrm{f}$ & 257 & 48,76 \\
& Cúbica & $\mathrm{w}=-507,355+29,7049 \times \mathrm{T}^{3}+4346,88 \times \mathrm{f}$ & 255 & 52,47 \\
& Exponencial & $\mathrm{w}=-142,146+440,234 \times e^{(\mathrm{T})} \times \mathrm{f}$ & 159 & 80,02 \\
\hline \multirow{5}{*}{ GC2015 } & Lineal & $\mathrm{w}=26,0895+32,0062 \times \mathrm{T}+275,0 \times \mathrm{f}$ & 9.60 & 84,65 \\
& Polinomial & $\mathrm{w}=478235+8,8315 \times \mathrm{T}^{2}+274,0 \times \mathrm{f}$ & 8,12 & 88,52 \\
& Cúbica & $\mathrm{w}=69,8528+2,74078 \times \mathrm{T}^{3}+1145,83 \times \mathrm{f}^{2}$ & 9,09 & 87,27 \\
& Exponencial & $\mathrm{w}=80,5152+33,7093 \times e^{(\mathrm{T})} \times \mathrm{f}$ & 5,04 & 96,32 \\
\hline
\end{tabular}

\section{Agradecimientos}

Los autores agradecen a la SEP por proporcionar la beca posgraduada de investigación en la Universidad Autónoma de Nuevo León (UANL) en México. Se le agradece al Centro de Investigación e Innovación en Ingeniería Aeronáutica por el apoyo financiero y tecnológico, además, por todas las facilidades brindadas para el desarrollo del trabajo de investigación. Se agradece al Departamento de Ciencias Técnicas de la Universidad de Granma por el apoyo brindado.

\section{Referencias}

[1] I. Asiltürk and S. Neseli, "Multi response optimisation of CNC turning parameters via taguchi method-based response surface analysis," Measurement, vol. 45, no. 4, pp. 785-794, 2012.

[2] A. E. Diniz, Á. R. Machado, and J. G. Corrêa, "Tool wear mechanisms in the machining of steels and stainless steels," The International Journal of Advanced Manufacturing Technology, vol. 87, no. 9, pp. 3157-3168, 2016.

[3] O. Pereira, A. Rodríguez, A. Fernández-Abia, J. Barreiro, and L. L. de Lacalle, "Cryogenic and minimum quantity lubrication for an eco-efficiency turning of \{AISI\} 304," Journal of Cleaner Production, vol. 139, pp. 440-449, 2016.
[4] S. E. Cordes, "Thermal stability of $\gamma$-alumina $\{\mathrm{PVD}\}$ coatings and analysis of their performance in machining of austenitic stainless steels," $\{C I R P\}$ Journal of Manufacturing Science and Technology, vol. 5, no. 1, pp. 20-25, 2012.

[5] A. Shokrani, V. Dhokia, and S. Newman, "Environmentally conscious machining of difficult-tomachine materials with regard to cutting fluids," International Journal of Machine Tools and Manufacture, vol. 57, pp. 83-101, 2012.

[6] M. Nalbant and Y. Yildiz, "Effect of cryogenic cooling in milling process of AISI 304 stainless steel," Transactions of Nonferrous Metals Society of China, vol. 21, no. 1, pp. 72-79, 2011.

[7] S. Saketi, J. Östby, and M. Olsson, "Influence of tool surface topography on the material transfer tendency and tool wear in the turning of 3161 stainless steel," Wear, vol. 368-369, pp. 239-252, 2016.

[8] Sandvik, "Torneado general coromant, s.: A4-a54," 2012.

[9] Tool-life testing with single-point turning tools. B94.55M., ANSI/ASME Std., 1985.

[10] G. Bartarya and S. Choudhury, "State of the art in hard turning," International Journal of Ma- 
chine Tools and Manufacture, vol. 53, no. 1, pp. 1-14, 2012.

[11] D. Jianxin, Z. Jiantou, Z. Hui, and Y. Pei, "Wear mechanisms of cemented carbide tools in dry cutting of precipitation hardening semi-austenitic stainless steels," Wear, vol. 270, no. 7, pp. 520 527, 2011.

[12] R. Suresh, S. Basavarajappa, V. Gaitonde, and G. Samuel, "Machinability investigations on hardened \{AISI\} 4340 steel using coated carbide insert," International Journal of Refractory Metals and Hard Materials, vol. 33, pp. 75-86, 2012.
[13] N. I. Galanis and D. E. Manolakos, "Surface roughness prediction in turning of femoral head," The International Journal of Advanced Manufacturing Technology, vol. 51, no. 1, pp. 79-86, 2010.

[14] A. Hamdan, A. A. D. Sarhan, and M. Hamdi, "An optimization method of the machining parameters in high-speed machining of stainless steel using coated carbide tool for best surface finish," The International Journal of Advanced Manufacturing Technology, vol. 58, no. 1, pp. 81-91, 2012. 